\title{
Resonant Photoelectron Imaging of Deprotonated Uracil Anion via Vibrational Levels of a Dipole-Bound Excited State
}

\author{
Dao-Ling Huang, ${ }^{\mathrm{a}}$ Hong-Tao Liu, ${ }^{\mathrm{b}}$ Chuan-Gang Ning, ${ }^{\mathrm{c}}$ Phuong Diem Dau, ${ }^{\mathrm{a}, \mathrm{H}}$ Lai-Sheng Wang ${ }^{\mathrm{a}, *}$ \\ ${ }^{a}$ Department of Chemistry, Brown University, Providence, Rhode Island 02912, USA \\ ${ }^{\mathrm{b}}$ Shanghai Institute of Applied Physics, Chinese Academy of Sciences, Shanghai 201800, China \\ 'Department of Physics, State Key Laboratory of Low-Dimensional Quantum Physics, Tsinghua University, Beijing \\ 100084, China
}

\begin{abstract}
We report both non-resonant and resonant high-resolution photoelectron imaging of cryogenically-cooled deprotonated uracil anions, N1[U-H] $]^{-}$, via vibrational levels of a dipole-bound excited state. Photodetachment spectroscopy of $\mathrm{N} 1[\mathrm{U}-\mathrm{H}]^{-}$was reported previously [H.T. Liu et al., Angew. Chem. Int. Ed. 53 (2014) 2464], in which forty-six vibrational autodetachment resonances due to the excited dipole-bound state were observed. By tuning the detachment laser to the vibrational levels of the dipole-bound state, we obtained high-resolution resonant photoelectron spectra, which are highly nonFranck-Condon. The resonant photoelectron spectra reveal many Franck-Condon inactive vibrational modes, significantly expanding the capability of photoelectron spectroscopy. A total of twenty one fundamental vibrational frequencies for the $\mathrm{N} 1[\mathrm{U}-\mathrm{H}] \cdot$ radical are obtained, including all eight lowfrequency out-of-plane modes, which are forbidden in non-resonant photoelectron spectroscopy. Furthermore, the breakdown of the $\Delta \mathrm{v}=-1$ propensity rule is observed for autodetachment from many vibrational levels of the dipole-bound state, due to anharmonic effects. In particular, we have observed intramolecular electron rescattering in a number of resonant photoelectron spectra, leading to excitations of low-frequency vibrational modes. Further theoretical study may be warranted, in light of the extensive experimental data and new observations, to provide further insight into the autodetachment dynamics and vibronic coupling in dipole-bound states, as well as electron molecule interactions.
\end{abstract}

KEY WORDS: dipole-bound state, deprotonated uracil, photoelectron imaging, resonant photoelectron spectroscopy, autodetachment.

\footnotetext{
*Corresponding author: E-mail Address: Lai-Sheng Wang@brown.edu

"Present address: Chemical Sciences Division, Lawrence Berkeley National Laboratory, Berkeley, California, 94720, USA
} 


\section{Introduction}

Photodetachment spectroscopy near the detachment threshold of cryogenically-cooled deprotonated uracil anions $\left(\mathrm{N} 1[\mathrm{U}-\mathrm{H}]^{-}\right)$was reported previously in the wavelength range from $357 \mathrm{~nm}$ to $335 \mathrm{~nm}$ [1]. Forty-six resonant peaks were observed as a result of autodetachment from vibrational levels of an excited dipole-bound state (DBS) with partial rotational resolution, as reproduced in Fig. 1. Photodetachment spectroscopy measures the total electron yield as a function of photon energy and was well demonstrated previously in observing autodetachment resonances [2-4], in particular, excited DBSs [5-7]. High-resolution data even provided information about rotational autodetachment from DBSs [8,9]. Photoelectron spectroscopy (PES) of anions involves measurement of the kinetic energies of photoelectrons and yields structural and spectroscopic information about the neutral final states. In principle, resonant PES could be obtained upon optical excitation to a resonant state of an anion, yielding information about the nature of the resonant state and the autodetachment process. However, most anion PES experiments are conducted with fixed wavelength detachment lasers. Relatively few resonant PES experiments on anions have been reported [10-12].

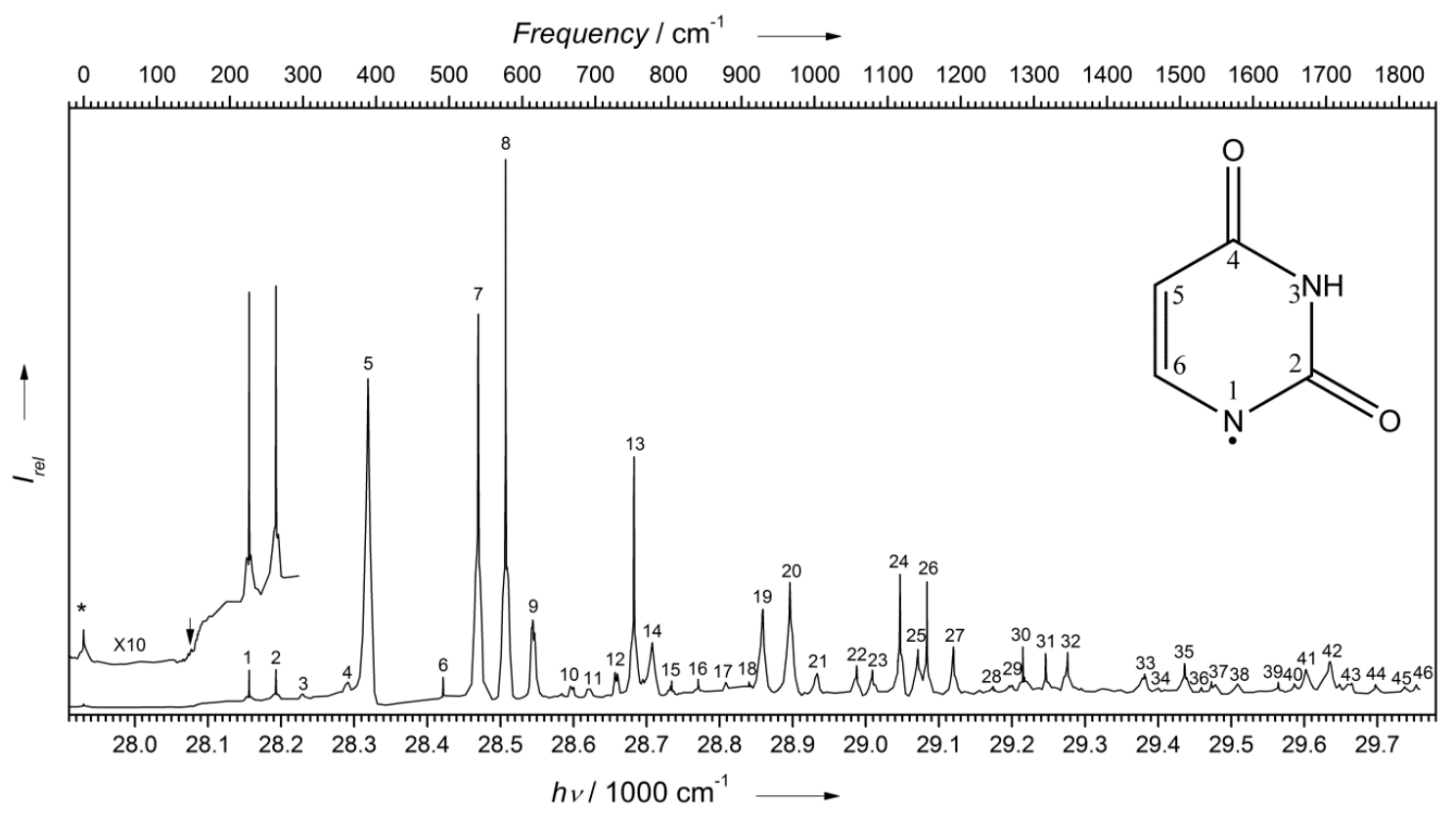

Fig. 1. Photodetachment spectrum of cryogenically-cooled deprotonated uracil anions, N1[U-H $]^{-}$, by measuring the total electron yield as a function of laser wavelengths across the detachment threshold. This figure is reproduced from Figure 1 in ref. 1 for the purpose of discussion. The arrow at $28076 \mathrm{~cm}^{-1}$ indicates the detachment threshold or the electron affinity of the $\mathrm{N} 1[\mathrm{U}-\mathrm{H}] \cdot$ radical, whose structure is shown in the inset. 
The first resonant PES via DBS was reported for the phenoxide anion [13], in which several FranckCondon inactive vibrational modes were observed. More importantly, we have shown that resonant PE spectra are extremely useful for assignments of the photodetachment spectra, because the autodetaching vibrational levels are revealed prominently in resonant PES [13,14]. This is especially important for spectral regions with high densities of vibrational states, where several energy levels may be accidentally degenerate $[15,16]$. Because the extra electron in the DBS only weakly interacts with the neutral core, there is very little structural change between the DBS and the neutral final state, resutling in the $\Delta v=-1$ vibrational propensity rule $[17,18]$ in the autodetachment via vibrational levels of the DBS. This propensity rule has led to mode-selective autodetachment [13-16], as well as to conformation-selective resonant PES [19].

By tuning the detachment laser to the vibrational resonances in Fig. 1, resonant PE spectra were obtained for $\mathrm{N} 1[\mathrm{U}-\mathrm{H}]^{-}$and were used for the assignments of the photodetachment spectrum previously [1]. In the current work, we focus on the detailed resonant PE imaging study and how the resonant PE spectra are used for the spectral assignments. The forty-six vibrational resonances in Fig. 1 contained fundamental and combinational modes of the DBS, as well as overlapping vibrational levels. While only a few Franck-Condon active vibrational peaks are observed in the non-resonant PE spectra, the resonant PE spectra result in many more vibrational peaks for the N1[U-H] • radical, including many Franck-Condon inactive modes and out-of-plane modes, which are symmetry-forbidden. Violation of the $\Delta \mathrm{v}=-1$ vibrational propensity rule has been observed in a number of cases, due to anharmonic effects. Vibrational features have also been observed, which seem to derive from intramolecular inelastic rescattering of the autodetaching electron with the neutral core.

\section{Experimental method}

The experiment was carried out on a third-generation electrospray ionization (ESI) PES apparatus [20], equipped with a cryogenically-cooled ion trap [21] and a high-resolution PE imaging lens [22]. Briefly, the $\mathrm{N} 1[\mathrm{U}-\mathrm{H}]^{-}$anions were produced by electrospray of a uracil solution in a mixed methanol/water solvent (9:1 volume ratio) adjusted to a $\mathrm{pH}$ of $\sim 8$ by adding $\mathrm{NaOH}$. The anions were 
transferred to the cryogenic ion trap, operated at $4.4 \mathrm{~K}$, by a set of quadrupole and octupole ion guides. The anions were accumulated and thermally cooled by a $\mathrm{He} / \mathrm{H}_{2}$ background gas in the ion trap for $0.1 \mathrm{~s}$ before being pulsed out into the extraction zone of a time-of-flight mass spectrometer. The N1[U-H] anions were mass-selected and focused into the interaction zone of a co-linear velocity map imaging system [22], where they were detached by selected wavelengths from a Nd:YAG pumped dye laser $(\Delta \lambda \sim$ $0.0015 \mathrm{~nm}$, Sirah Cobra-Stretch). Photoelectrons were accelerated and projected onto a set of 75-mm diameter micro-channel plates coupled to a phosphor screen. The photoelectron images were captured by a charge-coupled device camera and sent to a computer for data accumulation. The PE images were symmetrized and inverse-Abel transformed to obtain three-dimensional photoelectron distributions using pBASEX [23]. The imaging system was calibrated with PE images of $\mathrm{Au}^{-}$at several photon energies [24]. The PE spectral resolution was $3.8 \mathrm{~cm}^{-1}$ for electrons with $55 \mathrm{~cm}^{-1}$ kinetic energy (KE) and about $1.5 \%(\Delta \mathrm{KE} / \mathrm{KE})$ for $\mathrm{KE}$ above $1 \mathrm{eV}[13,25]$.
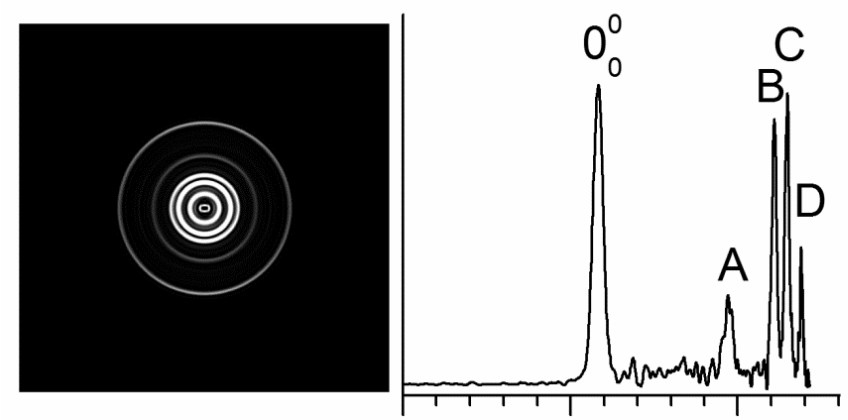

(a) $348.17 \mathrm{~nm}$
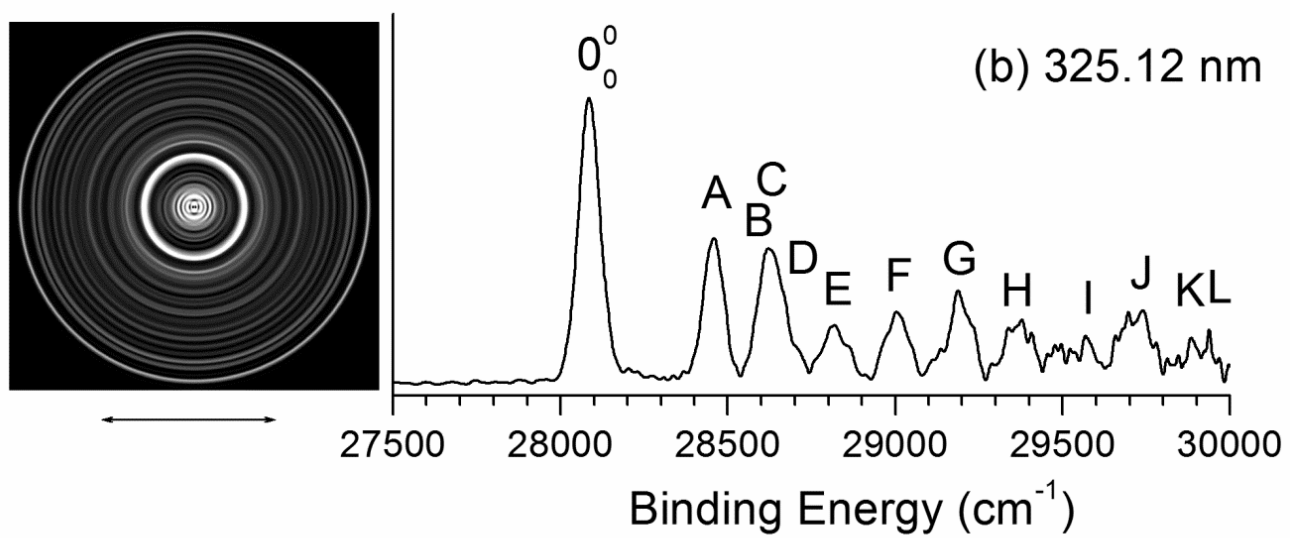

Fig. 2. Non-resonant photoelectron images and spectra of N1[U-H $]^{-}$at (a) $348.17 \mathrm{~nm}$ and (b) $325.12 \mathrm{~nm}$ and $4.4 \mathrm{~K}$ ion trap temperature. The double arrow below the images indicates the direction of the laser polarization. 


\section{Results}

\subsection{Non-resonant photoelectron images and spectra}

Fig. 2 shows the non-resonant PE images and spectra of $\mathrm{N} 1[\mathrm{U}-\mathrm{H}]^{-}$at two photon energies. The $325.12 \mathrm{~nm}$ spectrum displays numerous vibrational peaks (labeled by capital letters from $A$ to $L$ ) for the ground electronic state of the neutral N1[U-H]• radical. These peaks should represent a normal FranckCondon distribution, governed by the geometry changes between the anion and neutral ground state. The $348.17 \mathrm{~nm}$ spectrum is better resolved for the lower binding features. In particular, the three peaks labeled as $B, C$, and $D$ are well resolved; the peak width of the near-threshold peak $D$ is measured to be about 9 $\mathrm{cm}^{-1}$. In addition, there seem to be a threshold enhancement for these three peaks. The electron binding energies of all the observed vibrational peaks, their shifts from the $0_{0}^{0}$ transition, and the assignments are summarized in Table 1. The assignments are only made for peaks $A-D$ and their binding energies are measured from the high-resolution spectrum in Fig. 2a. The binding energies for peaks $E$ to $L$ are only given approximately from the low-resolution spectrum (Fig. 2b), because they each may contain overlapping vibrational features. Indeed, many more vibrational features are observed in the highresolution and resonant PE spectra to be presented next. In addition, the PE image in Fig. 2a shows that the photoelectron angular distribution (PAD) of the $0_{0}^{0}$ peak has $s+d$ character while that in Fig. $2 \mathrm{~b}$ is more isotropic, indicating that the PAD is kinetic energy dependent and the highest occupied molecular orbital (HOMO) of $\mathrm{N} 1[\mathrm{U}-\mathrm{H}]^{-}$is a $p$-type orbital $[26,27]$. 
Table 1 Observed binding energies (BE), shifts from the $0_{0}^{0}$ transition, and assignments of the resolved vibrational peaks from both non-resonant and resonant photoelectron spectra of $\mathrm{N} 1[\mathrm{U}-\mathrm{H}]^{-}$. The labels $0_{0}^{0}$ and $A-L$ correspond to those observed in the non-resonant spectra. Peaks $a-\mu$ are from the resonant spectra.

\begin{tabular}{|c|c|c|c|}
\hline Peak & $\overline{B E E}\left(\mathrm{~cm}^{-1}\right)^{\mathrm{a}}$ & Shifts $\left(\mathrm{cm}^{-1}\right)$ & Assignment \\
\hline $0_{0}^{0}$ & $28076(5)$ & 0 & neutral ground state \\
\hline$A$ & $28467(5)$ & 391 & $19^{1}$ \\
\hline$B$ & $28620(5)$ & 544 & $17^{1}$ \\
\hline$C$ & $28653(5)$ & 577 & $16^{1}$ \\
\hline$D$ & $28690(5)$ & 614 & $24^{1}$ \\
\hline$E$ & $\sim 28817$ & 741 & \\
\hline$F$ & $\sim 29007$ & 931 & \\
\hline$G$ & $\begin{array}{l}29188 \\
\sim\end{array}$ & 1112 & \\
\hline$H$ & $\begin{array}{l}29373 \\
\end{array}$ & 1297 & \\
\hline$I$ & $\sim 29572$ & 1496 & \\
\hline$J$ & $\sim 29739$ & 1663 & \\
\hline$K$ & $\sim 29884$ & 1808 & \\
\hline$L$ & $\sim 29939$ & 1863 & \\
\hline$a$ & $28190(5)$ & 114 & $27^{1}$ \\
\hline$b$ & $28236(8)$ & 156 & $26^{1}$ \\
\hline$c$ & $28301(5)$ & 225 & $27^{2}$ \\
\hline$d$ & $28377(8)$ & 301 & $26^{2}$ \\
\hline$e$ & $28437(5)$ & 361 & $25^{1}$ \\
\hline$f$ & $28759(8)$ & 683 & $19^{1} 26^{2}$ \\
\hline$g$ & $28830(5)$ & 754 & $15^{1}$ \\
\hline$h$ & $29008(7)$ & 932 & $19^{1} 17^{1}$ \\
\hline$i$ & $29046(8)$ & 970 & $13^{1}$ \\
\hline$j$ & $29359(8)$ & 1283 & $10^{1}$ \\
\hline$k$ & $28855(9)$ & 779 & $19^{2}$ \\
\hline$l$ & $29232(8)$ & 1156 & $16^{2}$ \\
\hline$m$ & $29496(10)$ & 1420 & $8^{1}$ \\
\hline$n$ & $29270(8)$ & 1194 & $24^{1} 16^{1}$ \\
\hline$o$ & $29196(8)$ & 1120 & $17^{1} 16^{1}$ \\
\hline$p$ & $29346(8)$ & 1268 & $22^{1} 17^{1}$ \\
\hline$q$ & $29430(8)$ & 1354 & $19^{1} 26^{2} 23^{1}$ \\
\hline$r$ & $29521(8)$ & 1451 & $6^{1}$ \\
\hline$s$ & $28736(8)$ & 660 & $17^{1} 27^{1}$ \\
\hline$t$ & $28986(8)$ & 910 & $14^{1}$ \\
\hline$u$ & 29134(8) & 1058 & $12^{1}$ \\
\hline$v$ & $29396(5)$ & 1320 & $9^{1}$ \\
\hline$w$ & $28806(8)$ & 730 & $22^{1}$ \\
\hline$x$ & $28553(10)$ & 477 & $27^{1} 25^{1}$ \\
\hline$y$ & $28960(8)$ & 884 & $18^{1} 19^{1}$ \\
\hline$z$ & $28592(8)$ & 516 & $26^{1} 25^{1}$ \\
\hline$\alpha$ & $28344(8)$ & 268 & $26^{1} 27^{1}$ \\
\hline$\beta$ & $28414(10)$ & 338 & $27^{3}$ \\
\hline$\gamma$ & $28529(10)$ & 453 & $26^{3}$ \\
\hline$\delta$ & $28572(10)$ & 496 & $18^{1}$ \\
\hline$\varepsilon$ & $28451(8)$ & 375 & $27^{2} 26^{1}$ \\
\hline$\zeta$ & $29240(8)$ & 1164 & $\mathrm{~b}$ \\
\hline$\eta$ & $29453(7)$ & 1377 & $\mathrm{~b}$ \\
\hline$\theta$ & $29280(9)$ & 1204 & b \\
\hline$\lambda$ & $29115(12)$ & 1035 & $18^{1} 17^{1}$ \\
\hline$\mu$ & $28905(9)$ & 829 & \\
\hline
\end{tabular}

${ }^{a}$ Numbers in parentheses indicate the experimental uncertainties in the last digit. 


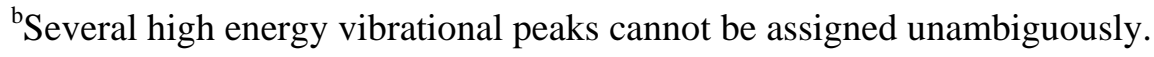

\subsection{Resonant photoelectron images and spectra}

By tuning the detachment laser to the vibrational resonances observed in the photodetachment spectrum shown in Fig. 1, resonantly enhanced PE spectra are obtained, as shown in Figs. 3-6. These spectra are organized according to the nature of the autodetaching vibrational levels of the DBS. The spectra in Fig. 3 correspond to excitation of a single vibrational mode of the DBS; those in Fig. 4 represent excitations to combinational vibrational levels of the DBS; Fig. 5 shows autodetachment from overlapping vibrational levels of the DBS, and Fig. 6 presents autodetachment from vibrational levels showing breakdown of the $\Delta \mathrm{v}=-1$ propensity rule. Numerous Franck-Condon inactive modes are observed in the resonant PE spectra and are labeled by lower case letters in Figs. 3-6. The labels in bold face in each spectrum indicate the final vibrational states of autodetachment, which are resonantly enhanced. All the observed binding energies and assignments of the additional features observed in the resonant PE spectra are also given in Table 1. Many of these peaks are observed in multiple spectra and the binding energies given in Table 1 are obtained from the most accurate measurements, i.e., from spectra in which a given vibrational feature has the lowest kinetic energy. 

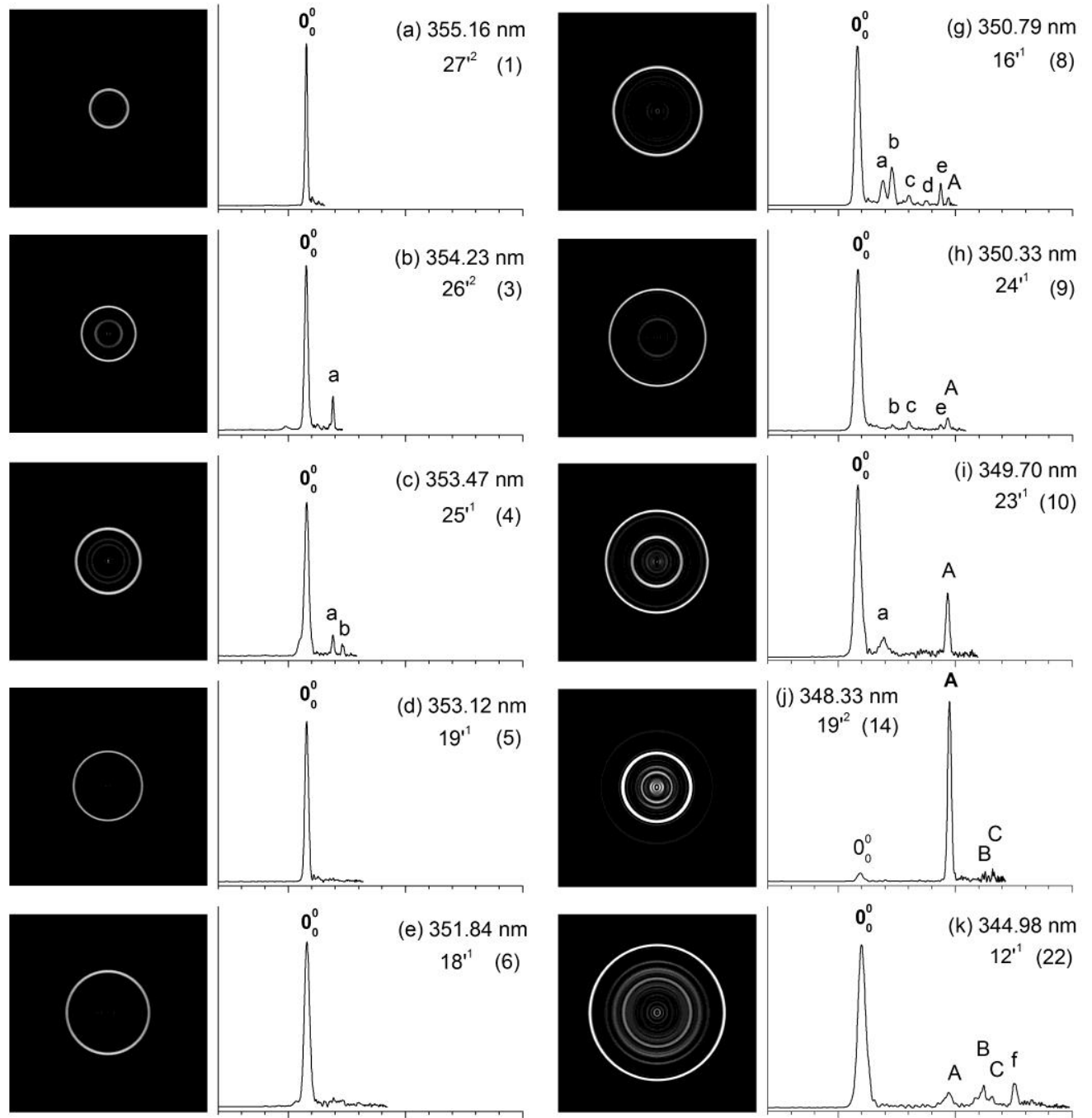

(j) $348.33 \mathrm{~nm}$ $19^{\prime 2}$ (14)
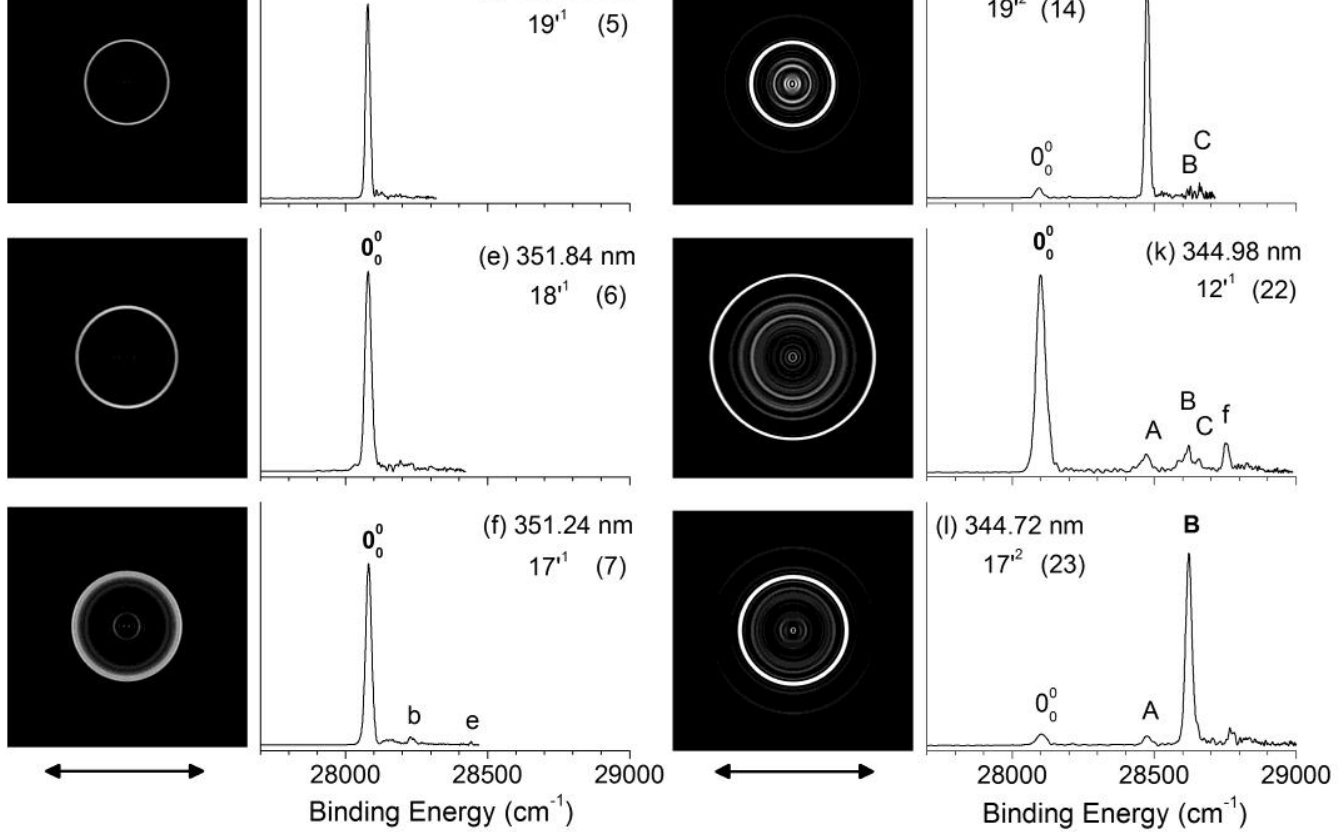

Fig. 3. Resonant photoelectron images and spectra of $\mathrm{N} 1[\mathrm{U}-\mathrm{H}]^{-}$at twelve different detachment wavelengths, corresponding to excitations to vibrational levels of single modes of the DBS. The resonant vibrational levels and the peak numbers (given in parentheses) according to Fig. 1 are labeled in each spectrum. The double arrows below the images indicate the direction of the laser polarization. The labels in capital letters are the same as in Fig. 2 and those in bold face indicate the autodetachment-enhanced final vibrational states. 

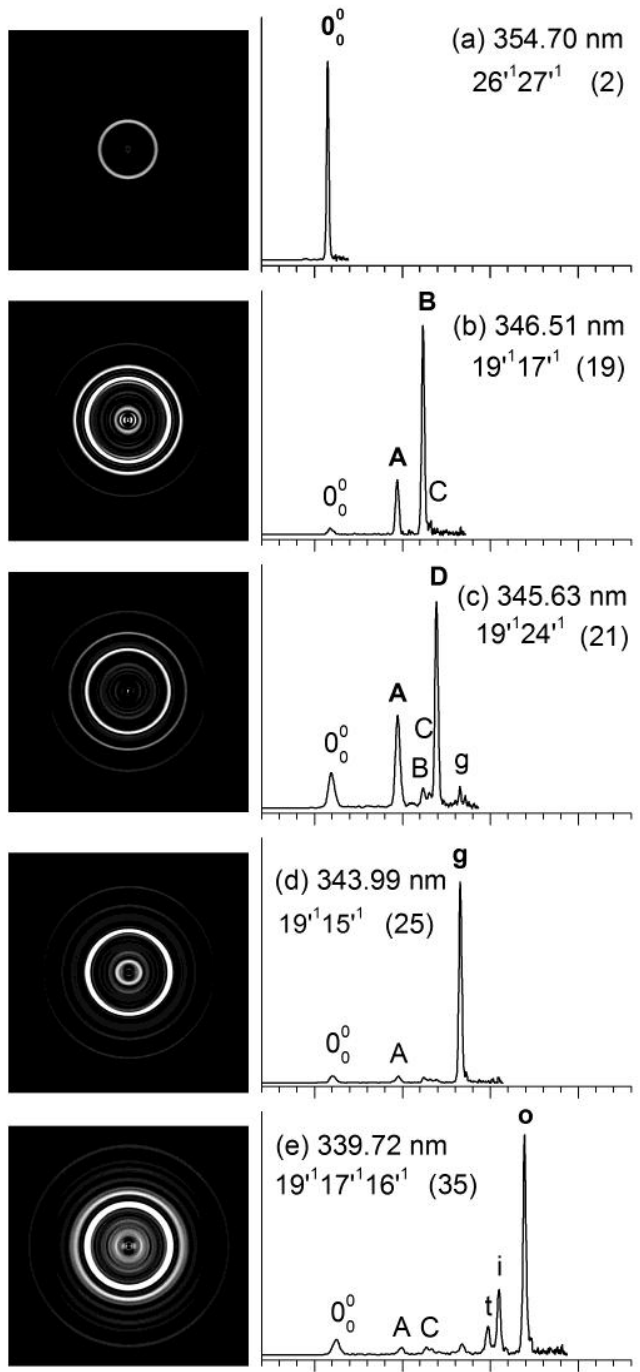

(e) $339.72 \mathrm{~nm}$ $19^{\prime 1} 17^{11} 16^{\prime 1}$ (35)
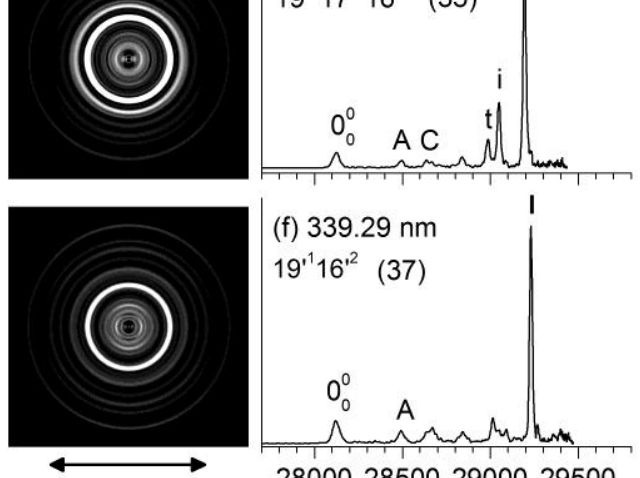

(f) $339.29 \mathrm{~nm}$ $19^{\prime 1} 16^{\prime 2}$ (37)

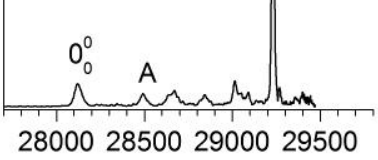
Binding Energy $\left(\mathrm{cm}^{-1}\right)$

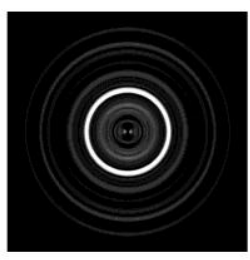

(g) $338.88 \mathrm{~nm}$

$19^{\prime 1} 16^{\prime 1} 24^{\prime 1}(38)$
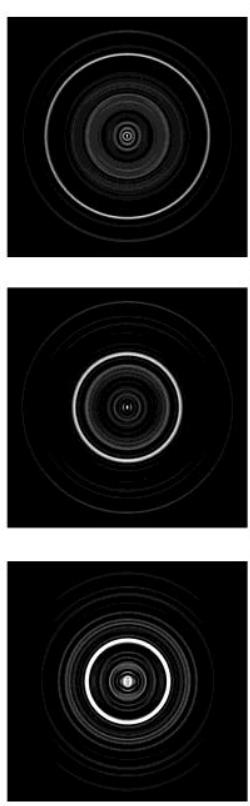
$19^{\prime 2} 26^{\prime 2} 23^{\prime 1}(43)$

C (h) $338.25 \mathrm{~nm}$ $12^{\prime 1} 16^{\prime 1}$ (39)

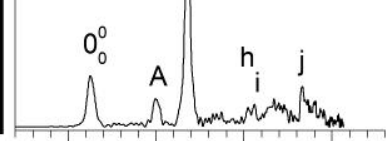

(i) $338.00 \mathrm{~nm}$ $17^{\prime 2} 16^{\prime 1}(40)$
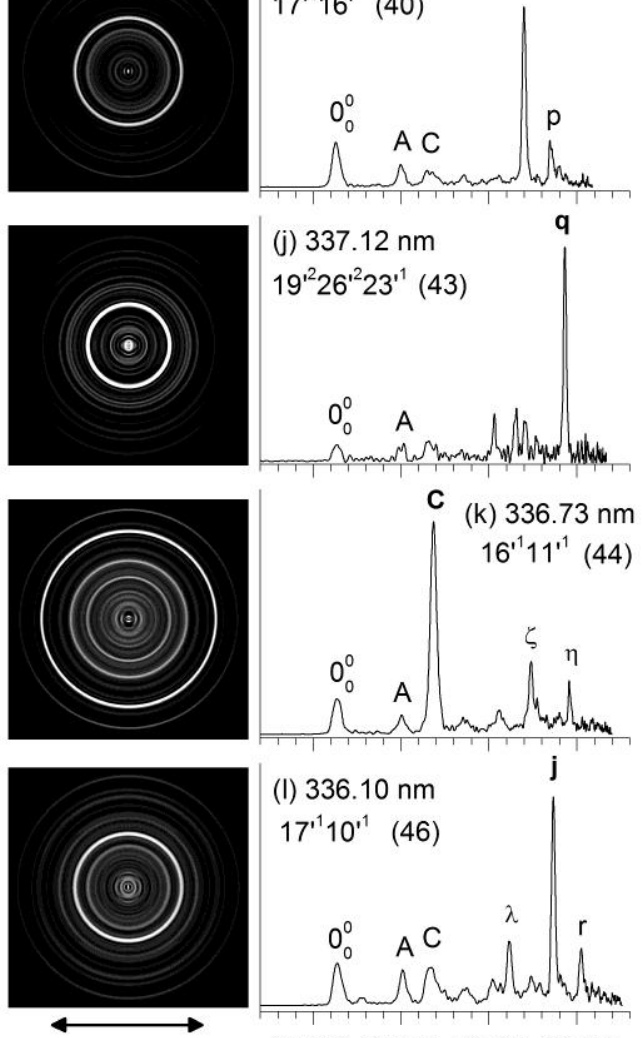

(I) $336.10 \mathrm{~nm}$

$17^{11} 10^{\prime 1} \quad(46)$

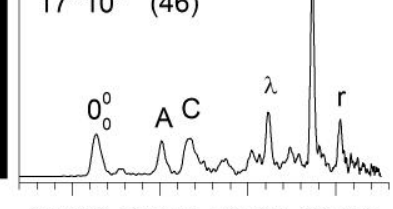

28000285002900029500

Binding Energy $\left(\mathrm{cm}^{-1}\right)$

Fig. 4. Resonant photoelectron images and spectra of $\mathrm{N} 1[\mathrm{U}-\mathrm{H}]^{-}$at twelve detachment wavelengths, corresponding to excitations to pure combinational vibrational levels of the DBS. The resonant vibrational levels and the peak numbers (given in parentheses) according to Fig. 1 are labeled in each spectrum. The double arrows below the images indicate the direction of the laser polarization. The labels in capital letters are the same as in Fig. 2 and those in bold face indicate the autodetachment-enhanced final vibrational states. 

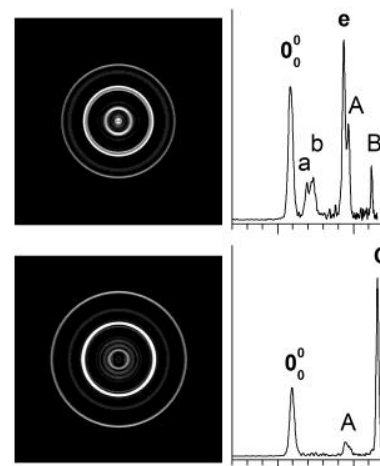

|
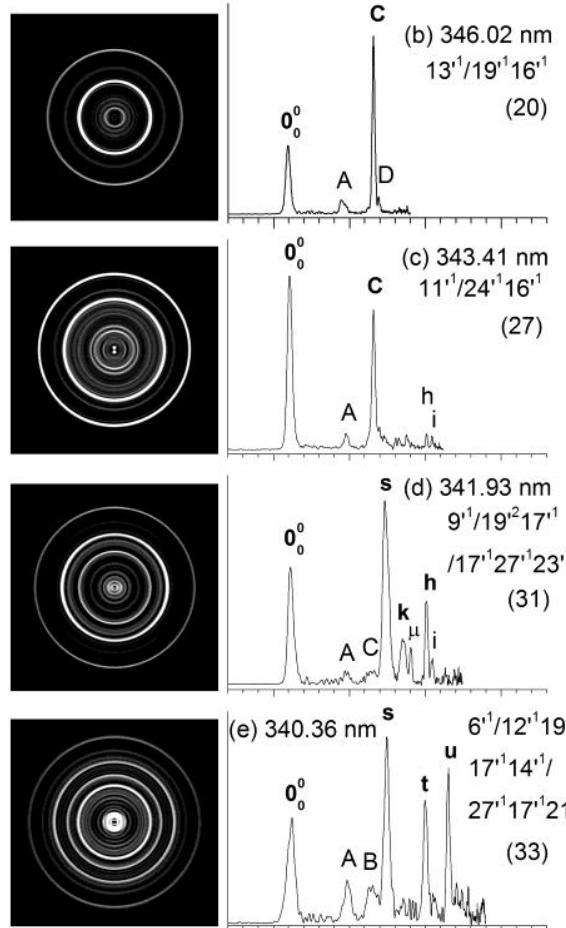

(e) $340.36 \mathrm{~nm}$

t u $17^{\prime 1} 14^{\prime 1} /$

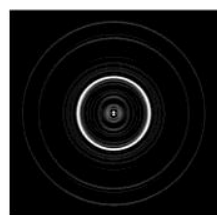

(f) $337.82 \mathrm{~nm}$ $5^{\prime^{1}} / 19^{\prime^{1} 10^{\prime 1}}$ (41)

\section{$\mathbf{0}_{0}^{0}$ \\ A}
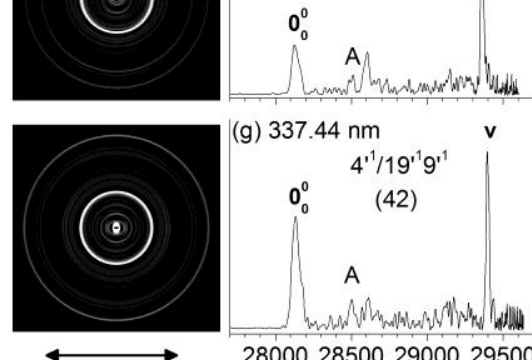

(g) $337.44 \mathrm{~nm}$

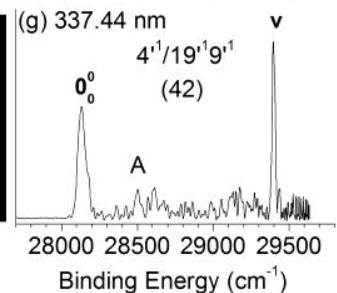

$6^{\prime 1 / 12} 2^{1} 19^{\prime 1}$

(33)
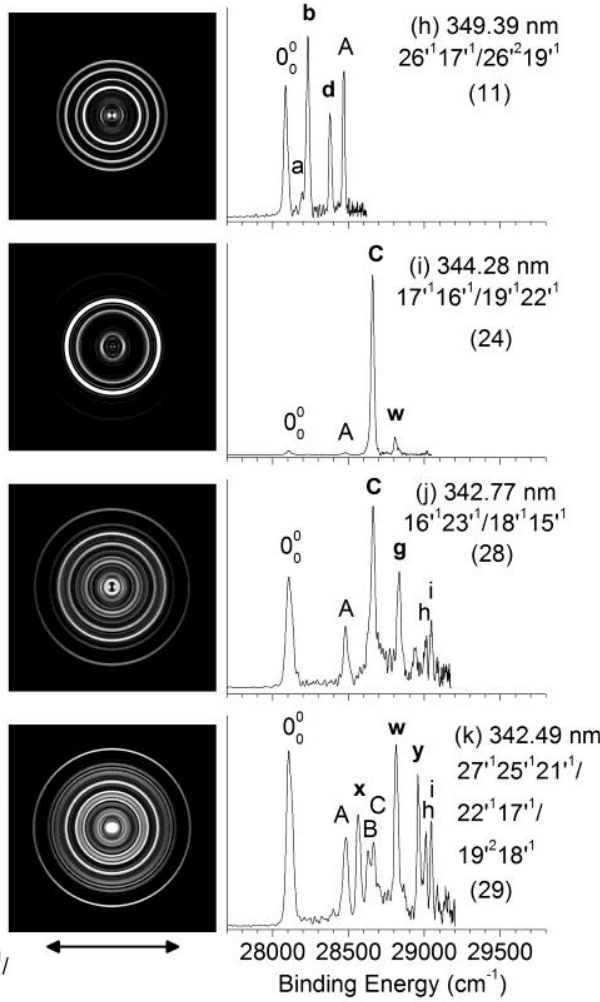

\section{.}

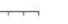$$
\text { . }
$$

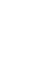



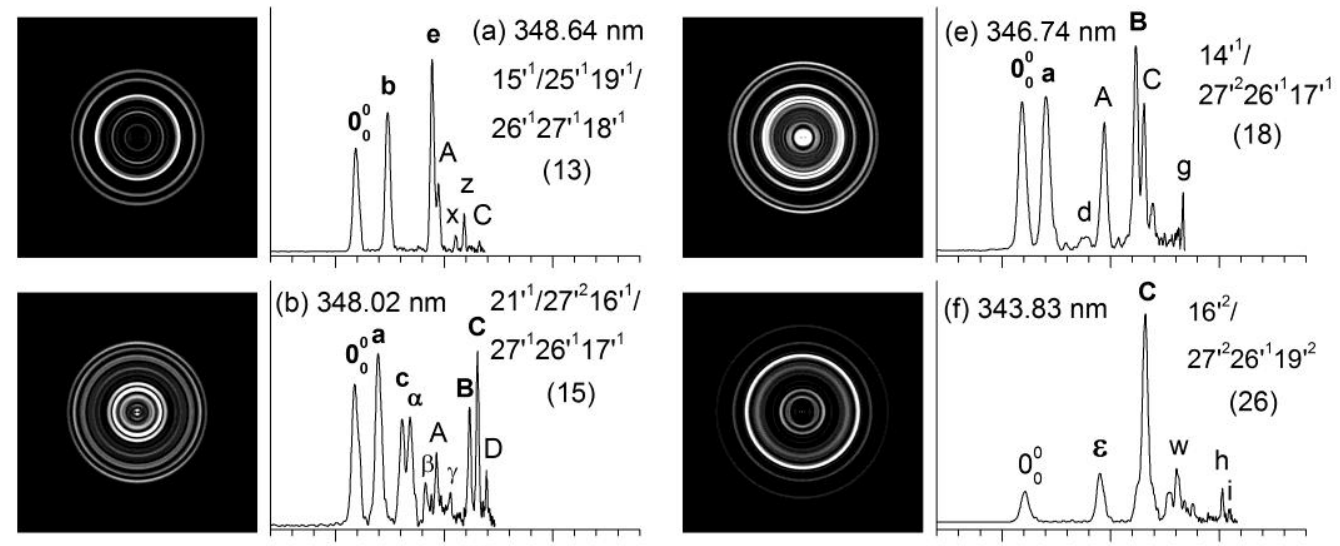

(b) $348.02 \mathrm{~nm} \quad 21^{1} 127^{\prime 2} 16^{\prime 1} /$
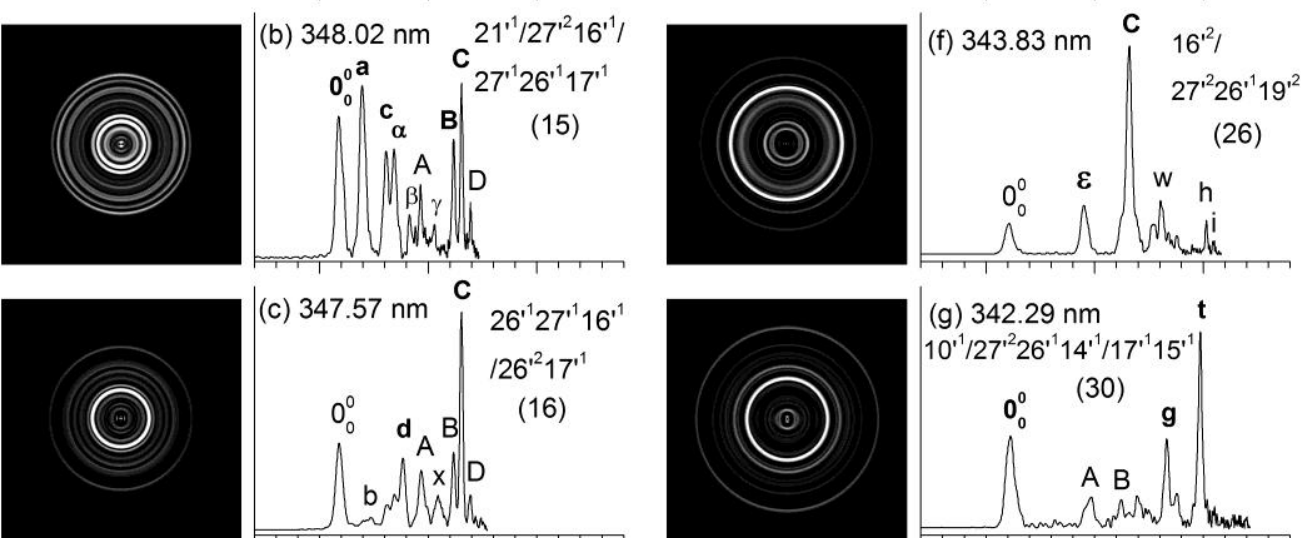

(c) $347.57 \mathrm{~nm}$

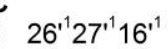
$126^{\prime 2} 17^{\prime 1}$

(16)
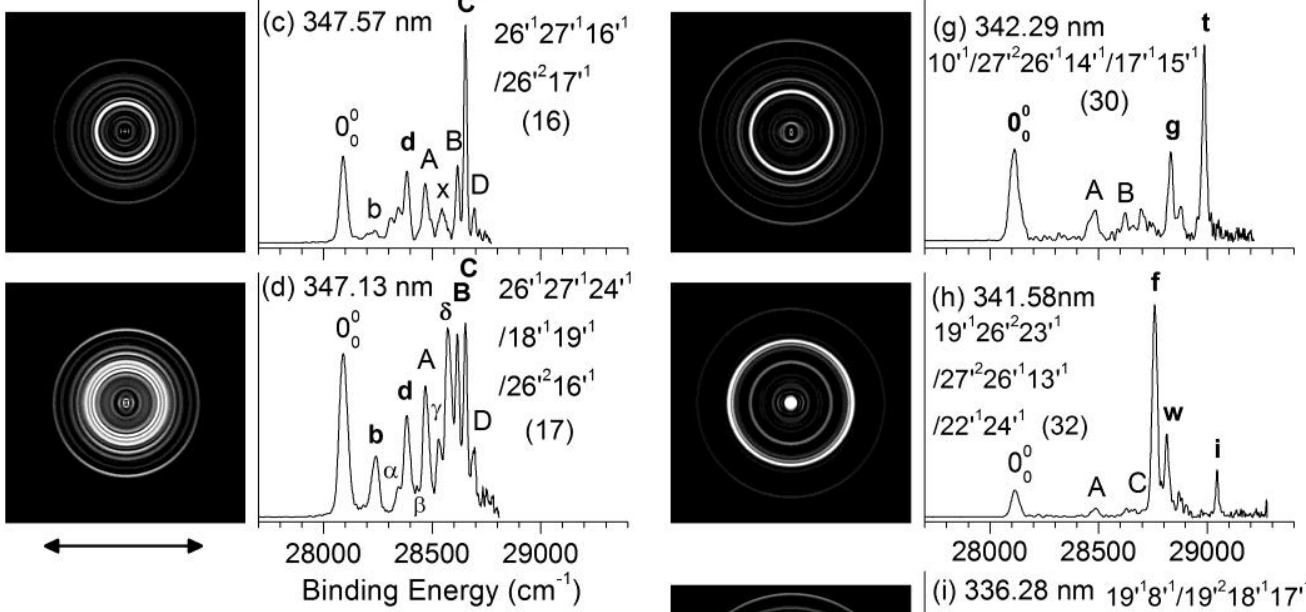

(h) $341.58 \mathrm{~nm}$

$19^{\prime 1} 26^{\prime 2} 23^{\prime 1}$

$127^{12} 26^{11} 13^{11}$

$122^{11} 24^{1}{ }^{1}(32)$
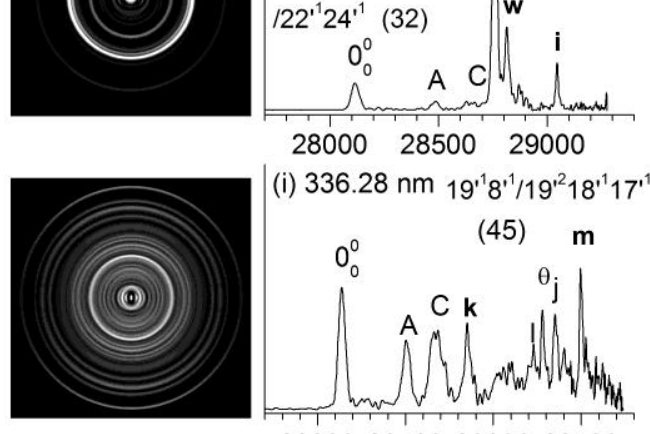

(i) $336.28 \mathrm{~nm} 19^{\prime^{1}} 8^{\prime^{1}} / 19^{\prime 2} 18^{\prime 1} 17^{\prime 1}$

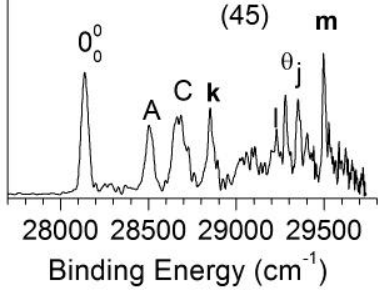

Fig. 6. Resonant photoelectron images and spectra of $\mathrm{N} 1[\mathrm{U}-\mathrm{H}]^{-}$at nine detachment wavelengths, corresponding to excitations to overlapping vibrational levels of the DBS. The resonant vibrational levels and the peak numbers (given in parentheses) according to Fig. 1 are labeled in each spectrum. All these spectra reveal autodetachment with the breakdown of $\Delta \mathrm{v}=-1$ propensity rule. The double arrows below the images indicate the direction of the laser polarization. The labels in capital letters are the same as in Fig. 2 and those in bold face indicate the autodetachment-enhanced final vibrational states.

The assignments of the vibrational peaks in the photodetachment spectrum (Fig. 1) were done previously using the resonant PE spectra and the $\Delta \mathrm{v}=-1$ propensity rule [1]. In the current study, each resonant PE spectrum is re-evaluated more thoroughly. In a number of cases involving overlapping 
Table 2 The observed photon energies, shifts from the ground vibrational level of the DBS, and the previous and current assignments of the vibrational resonances in Fig. 1.

\begin{tabular}{|c|c|c|c|c|}
\hline \multirow[t]{2}{*}{ peak } & \multirow{2}{*}{$\begin{array}{l}\text { Photon Energy }{ }^{\mathrm{a}} \\
\left(\mathrm{cm}^{-1}\right)\end{array}$} & \multirow{2}{*}{$\begin{array}{l}\text { Shifts } \\
\left(\mathrm{cm}^{-1}\right)\end{array}$} & \multicolumn{2}{|c|}{ Assignment } \\
\hline & & & Ref. 1 & Current \\
\hline 1 & $28156(3)$ & 226 & $27^{\prime 2}$ & $27^{\prime 2}$ \\
\hline 2 & 28193(3) & 263 & $27^{1} 26^{\prime 1}$ & $27^{\prime \prime} 26^{\prime 1}$ \\
\hline 3 & $28230(8)$ & 300 & $26^{\prime 2}$ & $26^{\prime 2}$ \\
\hline 4 & $28290(8)$ & 360 & $25^{\prime \prime}$ & $25^{\prime \prime}$ \\
\hline 5 & $28319(3)$ & 389 & $19^{\prime 1}$ & $19^{\prime \prime}$ \\
\hline 6 & $28422(3)$ & 492 & $18^{\prime \prime}$ & $18^{\prime \prime}$ \\
\hline 7 & $28470(3)$ & 540 & $17^{11}$ & $17^{\prime 1}$ \\
\hline 8 & $28507(3)$ & 577 & $16^{\prime 1}$ & $16^{\prime 1}$ \\
\hline 9 & $28545(5)$ & 615 & $24^{\prime \prime}$ & $24^{\prime 1}$ \\
\hline 10 & $28596(8)$ & 666 & $23^{\prime 1}$ & $23^{\prime 1}$ \\
\hline 11 & $28621(8)$ & 691 & $26^{\prime 2} 19^{\prime 1}$ & $26^{\prime 1} 17^{\prime \prime} / 26^{\prime 2} 19^{\prime 1}$ \\
\hline 12 & $28657(8)$ & 727 & $22^{\prime 1}$ & $22^{\prime 1} / 25^{\prime 2}$ \\
\hline 13 & $28683(3)$ & 753 & $15^{\prime 1}$ & $15^{\prime 1} / 25^{\prime 1} 19^{\prime 1} / 26^{\prime 1} 27^{\prime 1} 18^{\prime 1}$ \\
\hline 14 & $28708(5)$ & 778 & $19^{\prime 2}$ & $19^{\prime 2}$ \\
\hline 15 & $28734(5)$ & 804 & $\begin{array}{c}21^{\prime 1} / 27^{\prime 1} 26^{\prime 2} 19^{\prime 1} / 27^{\prime 2} 16^{\prime 1} \\
/ 27^{\prime 1} 26^{\prime 1} 17^{\prime 1}\end{array}$ & $21^{\prime 1} / 27^{\prime 2} 16^{\prime 1} / 27^{\prime 1} 26^{\prime 1} 17^{\prime 1}$ \\
\hline 16 & $28771(5)$ & 841 & $26^{2} 17^{1} / 26^{1} 17^{1} 16^{1}$ & $26^{\prime 1} 27^{\prime 1} 16^{\prime 1} / 26^{\prime 2} 17^{\prime 1}$ \\
\hline 17 & $28808(8)$ & 878 & $18^{\prime \prime} 19^{\prime 1} / 27^{\prime 1} 26^{\prime \prime} 24^{\prime 1}$ & $26^{\prime \prime} 27^{\prime} 24^{\prime 1} / 18^{\prime 1} 19^{\prime \prime} / 26^{\prime 2} 16^{\prime 1}$ \\
\hline 18 & $28840(8)$ & 910 & $14^{\prime \prime}$ & $14^{\prime \prime} / 27^{\prime 2} 26^{\prime \prime} 17^{\prime 1}$ \\
\hline 19 & $28859(3)$ & 929 & $19^{\prime 1} 17^{\prime \prime}$ & $19^{\prime \prime} 17^{\prime \prime}$ \\
\hline 20 & $28900(3)$ & 970 & $13^{\prime 1} / 19^{\prime 1} 16^{\prime 1}$ & $13^{\prime 1} / 19^{\prime 1} 16^{\prime 1}$ \\
\hline 21 & 28933(5) & 1003 & $27^{\prime 2} 19^{\prime 2} / 19^{\prime \prime} 24^{\prime \prime}$ & $19^{\prime \prime} 24^{\prime \prime}$ \\
\hline 22 & $28987(5)$ & 1057 & $12^{\prime \prime}$ & $12^{\prime 1}$ \\
\hline 23 & $29009(5)$ & 1079 & $26^{\prime 2} 19^{\prime 2} / 17^{\prime 2}$ & $17^{\prime 2}$ \\
\hline 24 & 29046(3) & 1116 & $19^{\prime \prime} 22^{\prime \prime} / 17^{\prime \prime} 16^{\prime \prime}$ & $17^{\prime \prime} 16^{\prime \prime} / 19^{\prime \prime} 22^{\prime \prime}$ \\
\hline 25 & 29071(3) & 1141 & $19^{\prime \prime} 15^{\prime 1}$ & $19^{\prime \prime} 15^{\prime 1}$ \\
\hline 26 & 29084(3) & 1154 & $11^{\prime \prime} / 16^{\prime 2}$ & $16^{\prime 2} / 27^{\prime 2} 26^{\prime 1} 19^{\prime 2}$ \\
\hline 27 & 29120(3) & 1190 & $19^{\prime 1} 21^{\prime \prime} / 24^{\prime 1} 16^{\prime 1}$ & $11^{\prime 1} / 24^{\prime 1} 16^{\prime 1}$ \\
\hline 28 & 29174(8) & 1244 & $18^{\prime 1} 15^{\prime 1}$ & $23^{\prime \prime} 16^{\prime 1} / 18^{\prime 1} 15^{\prime 1}$ \\
\hline 29 & 29198(8) & 1268 & $18^{\prime 1} 19^{\prime 2}$ & $27^{\prime} 25^{\prime} 21^{\prime 1} / 22^{\prime 1} 17^{\prime 1} / 18^{\prime 1} 19^{\prime 2}$ \\
\hline 30 & $29215(3)$ & 1285 & $10^{\prime 1}$ & $10^{\prime 1} / 27^{\prime 2} 26^{\prime 1} 14^{\prime 1} / 17^{\prime 1} 15^{\prime 1}$ \\
\hline 31 & $29246(3)$ & 1316 & $9^{\prime 1} / 19^{\prime 1} 17^{\prime 1} / 27^{\prime 1} 26^{\prime 1} 12^{\prime 1}$ & $9^{\prime 1} / 19^{\prime 2} 17^{\prime 1} / 17^{\prime 1} 27^{\prime} 23^{\prime 1}$ \\
\hline 32 & 29276(3) & 1346 & $17^{\prime \prime} 21^{\prime \prime}$ & $19^{\prime 1} 26^{\prime 2} 23^{\prime 1} / 13^{\prime 1} 27^{\prime 2} 26^{\prime 1} / 22^{\prime 1} 24^{\prime \prime}$ \\
\hline 33 & $29381(8)$ & 1451 & $6^{\prime \prime} / 17^{\prime \prime} 14^{\prime \prime}$ & $6^{\prime 1} / 12^{\prime 1} 19^{\prime \prime} / 17^{\prime 1} 14^{\prime \prime} / 27^{\prime 1} 17^{\prime \prime} 21^{\prime \prime}$ \\
\hline 34 & $29400(8)$ & 1470 & $26^{\prime 2} 19^{\prime 3}$ & $b$ \\
\hline 35 & 29436(5) & 1506 & $19^{\prime 2} 22^{\prime 1}$ & $19^{\prime 1} 17^{\prime 1} 16^{\prime 1}$ \\
\hline 36 & 29459(8) & 1529 & $19^{\prime 2} 15^{\prime \prime}$ & \\
\hline 37 & $29473(8)$ & 1543 & $11^{\prime \prime} 19^{\prime 1}$ & $16^{\prime 2} 19^{\prime 1}$ \\
\hline 38 & $29509(8)$ & 1579 & $19^{\prime 2} 21^{\prime 1}$ & $19^{\prime \prime} 16^{\prime \prime} 24^{\prime 1}$ \\
\hline 39 & $29564(8)$ & 1634 & $18^{\prime 1} 15^{\prime 1} 19^{\prime \prime}$ & $12^{\prime \prime} 16^{\prime \prime}$ \\
\hline 40 & $29586(8)$ & 1656 & $18^{\prime 1} 19^{\prime 3}$ & $17^{\prime 2} 16^{\prime 1}$ \\
\hline 41 & $29602(8)$ & 1672 & $5^{\prime 1} / 10^{\prime 1} 19^{\prime 1}$ & $5^{\prime 1} / 10^{\prime \prime} 19^{\prime 1}$ \\
\hline 42 & $29635(8)$ & 1705 & $4^{\prime 1} / 9^{\prime 1} 19^{\prime 1}$ & $4^{\prime 1} / 9^{\prime 1} 19^{\prime 1}$ \\
\hline 43 & 29663(8) & 1733 & $17^{\prime} 21^{\prime \prime} 19^{\prime \prime}$ & $19^{\prime 2} 26^{\prime 2} 23^{\prime 1}$ \\
\hline 44 & $29697(8)$ & 1767 & $16^{\prime \prime} 21^{\prime \prime} 19^{\prime \prime}$ & $16^{\prime \prime} 11^{\prime 1}$ \\
\hline 45 & $29737(8)$ & 1807 & $8^{\prime 1} 19^{\prime \prime}$ & $8^{\prime 1} 19^{\prime 1} / 19^{\prime 2} 18^{\prime 1} 17^{\prime 1}$ \\
\hline 46 & $29753(8)$ & 1823 & $7^{\prime 1} 19^{\prime 1}$ & $17^{\prime 1} 10^{\prime 1}$ \\
\hline
\end{tabular}

${ }^{a}$ Numbers in parentheses indicate the experimental uncertainties in the last digit.

${ }^{\mathrm{b}}$ Peaks 34 and 36 were not re-evaluated. 
vibrational levels, reassignments are done, as compared in Table 2, where the observed photon energies of the vibrational resonances in Fig. 1, shifts from the ground vibrational level of the DBS, and the previous and current assignments are summarized. Table 3 reproduces the calculated and experimental vibrational frequencies of [N1-H] • from ref. 1 and the comparison with those obtianed in the current study. Only one vibrational frequency $\left(v_{11}\right)$ is improved in the current study due to the reassignment of peak 27 in the photodetachment spectrum (Fig. 1), as shown in Table 2.

Table 3 Comparison of theoretical and previous and current experimental vibrational frequencies (in $\mathrm{cm}^{-}$ ${ }^{1}$ ) of the dehydrogenated uracil radical $(\mathrm{N} 1[\mathrm{U}-\mathrm{H}] \bullet)$. The harmonic frequencies calculated with the B3LYP/6-311++G** method is from ref 1 .

\begin{tabular}{|c|c|c|c|c|c|}
\hline \multirow[t]{2}{*}{ mode } & \multirow[t]{2}{*}{ symmetry } & \multirow[t]{2}{*}{ theoretical } & \multicolumn{2}{|c|}{ experimental } & \multirow[t]{2}{*}{ peak $^{\mathrm{b}}$} \\
\hline & & & Previous $^{\mathrm{a}}$ & current & \\
\hline$v_{1}$ & $\mathrm{~A}^{\prime}$ & 3581 & & & \\
\hline$v_{2}$ & & 3222 & & & \\
\hline$v_{3}$ & & 3145 & & & \\
\hline$v_{4}$ & & 1713 & 1705 & 1705 & 42 \\
\hline$v_{5}$ & & 1694 & 1672 & 1672 & 41 \\
\hline$v_{6}$ & & 1469 & 1451 & 1451 & 33 \\
\hline$v_{7}$ & & 1437 & & & \\
\hline$v_{8}$ & & 1411 & & & \\
\hline$v_{9}$ & & 1342 & 1316 & 1316 & 31 \\
\hline$v_{10}$ & & 1301 & 1285 & 1285 & 30 \\
\hline$v_{11}$ & & 1186 & 1154 & $1190^{c}$ & 27 \\
\hline$v_{12}$ & & 1082 & 1057 & 1057 & 22 \\
\hline$v_{13}$ & & 982 & 970 & 970 & 20 \\
\hline$v_{14}$ & & 920 & 910 & 910 & 18 \\
\hline$v_{15}$ & & 757 & 753 & 753 & 13 \\
\hline$v_{16}$ & & 583 & 577 & 577 & 8 \\
\hline$v_{17}$ & & 545 & 540 & 540 & 7 \\
\hline$v_{18}$ & & 501 & 492 & 492 & 6 \\
\hline$v_{19}$ & & 392 & 389 & 389 & 5 \\
\hline$v_{20}$ & $\mathrm{~A}^{\prime \prime}$ & 980 & & & \\
\hline$v_{21}$ & & 803 & 804 & 804 & 15 \\
\hline$v_{22}$ & & 734 & 727 & 727 & 12 \\
\hline$v_{23}$ & & 684 & 666 & 666 & 10 \\
\hline$v_{24}$ & & 633 & 615 & 615 & 9 \\
\hline$v_{25}$ & & 357 & 360 & 360 & 4 \\
\hline$v_{26}$ & & 152 & 150 & 150 & \\
\hline$v_{27}$ & & 113 & 113 & 113 & \\
\hline
\end{tabular}

${ }^{a}$ Previous experimental frequencies are reproduced from Table 1 in ref. 1.

${ }^{\mathrm{b}}$ Peak numbers are according to the labels in Fig. 1.

${ }^{\mathrm{c}}$ This frequency is improved relative to that reported in ref. 1. 


\section{Discussion}

\subsection{Assignment of the non-resonant photoelectron spectra}

We used the calculated vibrational frequencies in Table 3 to assist the assignment of the observed vibrational structure $(A-D)$ in Fig. 2. Since the non-resonant PE spectra represent Franck-Condon-active vibrational modes for the ground state detachment transition, only symmetry-allowed modes are supposed to be observed. The previous calculation suggested that the $\mathrm{N} 1[\mathrm{U}-\mathrm{H}] \bullet$ radical has a planar structure with $C_{s}$ symmetry [1]. Hence, all in-plane vibrational modes (A') or even quanta of out-of-plane modes (A") are allowed. While we can readily assign peaks $A, B$, and $C$ to modes $v_{19}, v_{17}, v_{16}$, respectively, peak $D$ with the measured frequency of $614 \mathrm{~cm}^{-1}$ does not match any in-plane mode or even quanta of any out-ofplane mode. Thus, we tentatively assigned it to be the out-of-plane $v_{24}$ mode on the basis on the calculated vibrational frequency $\left(633 \mathrm{~cm}^{-1}\right)$, indicating that $\mathrm{N} 1[\mathrm{U}-\mathrm{H}] \bullet$ is not truly planar or somewhat floppy [1]. In fact, the $v_{24}$ mode was also observed in the photodetachment spectrum (peak 9 in Fig. 1 and Table 2) with the same vibrational frequency in the DBS. As will be discussed in the following Sections, all the four assigned fundamental modes are also observed in the resonant PE spectra. As mentioned above, peaks $E-$ $L$ in Fig. $2 \mathrm{~b}$ are broad and may not be assigned to a single vibrational level. Indeed, the high-resolution resonant PE spectra to be discussed next show that many of these peaks do contain multiple vibrational excitations.

\subsection{Autodetachment from single-mode vibrational levels of the DBS}

According to the $\Delta \mathrm{v}=-1$ vibrational propensity rule, the $n$th vibrational level of a given mode $\left(v_{x}^{\prime}\right)$ of the DBS is favored to autodetach to the $(n-1)$ vibrational level of the corresponding mode $\left(v_{x}\right)$ in the neutral final state, resulting in an enhanced peak for the $v_{x}^{(n-1)}$ vibrational level in a resonant PE spectrum with mode-selectivity [13-16]. Here we use a prime in $v_{x}^{\prime}$ to designate vibrational modes for the DBS and $v_{x}$ to designate those for the neutral final state. However, we have shown previously that the vibrational frequencies of the neutral final state and the DBS are the same within our experimental uncertainty $( \pm$ $5-10 \mathrm{~cm}^{-1}$ ), because of the weak perturbation of the DBS electron to the neutral core. 
In Figs. $3 \mathrm{c}-3 \mathrm{i}$ and $3 \mathrm{k}$, the $0_{0}^{0}$ peak is significantly enhanced, suggesting autodetachment from a fundamental excitation of a single vibrational mode $\left(v_{x}{ }^{1}\right)$ of the DBS of N1[U-H] $]^{-}$in each case. As indicated in each spectrum, the detachment photon energies used for Figs. 3c-3i and 3k correspond to resonant excitations to the following vibrational levels of the DBS, namely, $25^{\prime 1}, 19^{\prime 1}, 18^{\prime 1}, 17^{\prime 1}, 16^{\prime 1}, 24^{\prime 1}$, $23^{\prime 1}$, and $12^{\prime 1}$, respectively. However, the enhanced $0_{0}^{0}$ peak in Figs. $3 \mathrm{a}$ and $3 \mathrm{~b}$ appears to involve autodetachment from the overtones of modes $v_{27}$ and $v_{26}$, respectively, disobeying the $\Delta v=-1$ propensity rule. These observations suggest significant anharmonic effects in the two low-frequency modes [17]. Figs. $3 \mathrm{j}$ and 31 show that peak $A\left(19^{1}\right)$ and peak $B\left(17^{1}\right)$ are dramatically enhanced, indicating autodetachment from the overtones of $19^{\prime 2}$ and $17^{\prime 2}$, respectively, as labeled in each spectrum. It should also be pointed out that the PADs for all the autodetachment-enhanced peaks in Fig. 3 are isotropic, in contrast to that of the non-resonant PE image in Fig. 2a.

\subsection{The observation of intramolecular electron rescattering effects}

Interestingly, in addition to the significantly enhanced autodetachment peaks, other weak peaks, which are not present in the non-resonant spectra (Fig. 2), are observed in certain resonant spectra, such as peak $a$ in Fig. 3b, peaks $a$ and $b$ in Fig. 3c, peaks $b$ and $e$ in Fig. 3f, peaks $b, c$, and $e$ in Fig. 3h, and peak $a$ in Fig. 3i. In particular, Fig. 3g (corresponding to autodetachment from the $16^{\prime 1}$ level of the DBS of $\mathrm{N} 1[\mathrm{U}-\mathrm{H}]^{-}$) shows numerous weak peaks with significant intensities. Comparison with the non-resonant spectra reveals that these peaks are completely non-Franck-Condon, as shown in Fig. 7. The binding energies and assignments of these weak peaks are given in Table 1. By comparing with the calculated frequencies, these weak peaks can be readily assigned as $a\left(27^{1}\right), b\left(26^{1}\right), c\left(27^{2}\right), d\left(26^{2}\right)$, and $e\left(25^{1}\right)$, which are also labeled in Fig. 7. These correspond to the three lowest frequency modes for the N1[U-H]• final state (Table 3), and they are all out-of-plane modes and are completely inactive in the non-resonant PE spectra (Fig. 2).

The observation of these weak peaks suggests violation of mode-selectivity and the $\Delta v=-1$ propensity rule for vibrational autodetachment from DBS. We will use Fig. $3 g$ as an example for the following discussion. In this case, the $16^{\prime 1}$ level of the DBS is resonantly excited; the $\Delta v=-1$ propensity 
rule and mode selectivity would dictate that the final state of autodetachment should only be the ground vibrational state of the neutral, resulting in an enhanced $0_{0}^{0}$ peak. While the $0_{0}^{0}$ is indeed enhanced (Fig. 7), how do the $27^{1}, 26^{1}, 27^{2}, 26^{2}$, and $25^{1}$ final vibrational levels get excited?

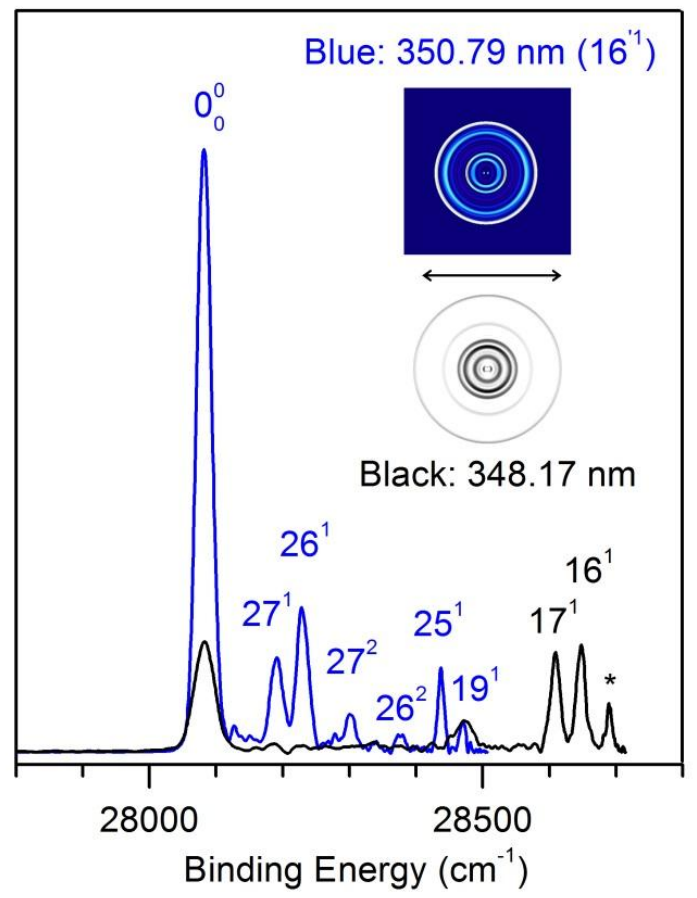

Fig. 7. Comparison of the resonant photoelectron image and spectrum at $350.79 \mathrm{~nm}$ (blue) from Fig. $3 \mathrm{~g}$ with the nonresonant photoelectron image and spectrum at $348.17 \mathrm{~nm}$ (black) from Fig. 2a. The double arrow indicates the polarization of the detachment laser. The two spectra are normalized by height using the $19^{1}$ peak. The resonant enhancement of the $0_{0}{ }^{0}$ peak and the appearance of non-Franck-Condon peaks are readily seen in the resonant spectrum.

There are two possible explanations for these observations. The first involves intramolecular vibrational energy redistribution (IVR) of the initially excited $16^{\prime 1}$ level in the DBS to other vibrational modes, followed by autodetachment from these modes. Among many other observations, IVR can be readily ruled out using simple energetic considerations. Simply consider the observation of the $25^{1}$ level, which would require autodetachment from the $25^{\prime 2}$ level of the DBS at $720 \mathrm{~cm}^{-1}$ above the ground vibrational level of the DBS $\left(v_{25}=360 \mathrm{~cm}^{-1}\right.$, Table 3$)$. This level cannot be reached from the $16^{\prime 1}$ initial excitation at $577 \mathrm{~cm}^{-1}$ (the vibrational frequency of $v_{16}$, Table 3).

A more likely explanation is intramolecular rescattering of the outgoing electron by the molecular core. As shown in Fig. 8 for the relevant energy levels, autodetachment from the $16^{\prime 1}$ level of the DBS 


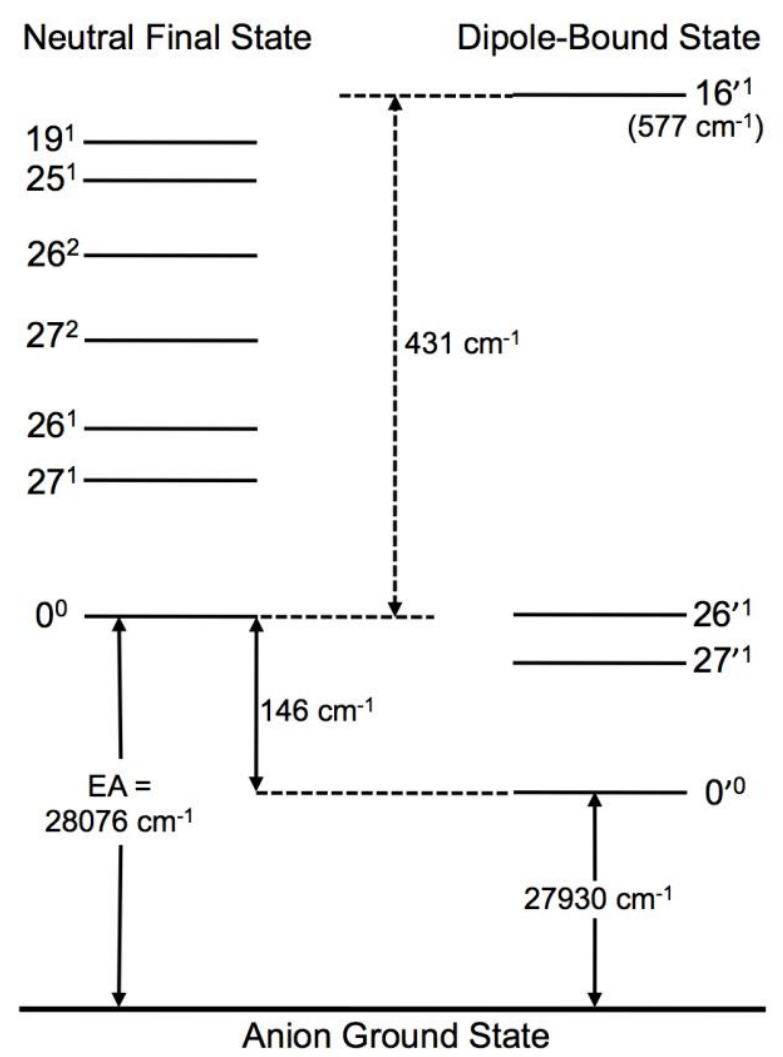

Fig. 8. Schematic energy level diagram showing selected vibrational levels of the dipole-bound excited state of $\mathrm{N} 1[\mathrm{U}-\mathrm{H}]^{-}$and those of the neutral N1[U-H] radical. The electron affinity (EA) of N1[U-H]•, the excitation energy to the ground vibrational level of the dipole-bound state, and the binding energy $\left(146 \mathrm{~cm}^{-1}\right)$ of the dipole-bound state are shown, as well as the electron kinetic energy $\left(431 \mathrm{~cm}^{-1}\right)$ from autodetachment from the $16^{1}$ vibrational of the dipole-bound state.

would result in an outgoing electron with a kinetic energy of $431 \mathrm{~cm}^{-1}$. There is some probability that this outgoing electron can be scattered inelastically with the neutral core, losing energy and causing vibrational excitations. This intramolecular rescattering can be viewed as a half-collision and is analogous to electron energy loss spectroscopy (EELS), which is an important vibraitonal spectroscopic techqniue to study surfaces and adsorbates on surfaces $[28,29]$. Similar intramolecular electron re-scattering effects have been invoked to interpret the observation of forbidden rotational transitions in resonant two-photon PES involving Rydberg states [30], as well as unexpected vibrational excitations in electron impact spectra of neutral molecules [31]. In fact, we have observed similar weak vibrational features in several recent resonant PE studies on involving DBS $[15,16]$, albeit none as extensive as in the current case.

Interestingly, the electron rescattering seems to depend on the vibrational modes of the DBSs from which the autodetachment occurs. For example, as displayed in Fig. 3, while autodetachment from $26^{\prime 2}$, 
$25^{\prime 1}, 17^{\prime 1}, 16^{\prime 1}, 24^{\prime 1}$, and $23^{\prime 1}$ shows the extra peaks due to electron rescattering, that from $19^{\prime 1}$ and $18^{\prime 1}$ does not give any extra peaks. There are also no such extra peaks in Figs. 3j-31. Hence, there are still unanswered questions about this phenomenon. Why does the autodetachment from the $16^{\prime 1}$ level give the most extensive rescattering effects? Is it related to the precise kinetic energy of the outgoing electron? The symmetry of the autodetaching vibrational levels seems to have no major effects, because rescattering is observed from both in-plane and out-of-plane autodetaching modes. It seems that further theoretical considerations are warranted, which should provide further sights into the vibronic coupling and autodetachment dynamics, as well as electron-molecule interactions.

\subsection{Autodetachment from combinational vibrational levels of the DBS}

As shown previously [13-16], the assignments of the resonant vibrational peaks above the neutral threshold in a photodetachment spectrum is usually based on resonant PE spectra. In the low frequency region, where the vibrational density of state is low and usually only one mode is involved, such as those in Fig. 3, the assignment of the resonant PE spectra is straightforward. However, for highly excited vibrational states of the DBS, where multiple modes or overlapping vibrational levels often occur, the complete assignments for the corresponding resonant PE spectra can be challenging.

In cases of combinational vibrational levels $\left(v_{x}{ }^{m} v_{y}{ }^{n} \ldots\right)$ of the DBS, according to the $\Delta \mathrm{v}=-1$ propensity rule and mode-selectivity, the final neutral level can be either $v_{x}{ }^{m-1} v_{y}{ }^{n} \ldots$ or $v_{x}{ }^{m} v_{y}{ }^{n-1} \ldots$, depending on the autodetachment rates of the different modes $\left(v_{x}^{\prime m} \rightarrow v_{x}{ }^{m-1}\right)$ or $\left(v_{y}{ }^{n} \rightarrow v_{y}{ }^{n-1}\right)$. However, Fig. 4a, which corresponds to excitation to the $26^{1} 27^{1}$ combinational level of the DBS, shows an enhanced $0_{0}^{0}$ peak, disobeying the $\Delta v=-1$ propensity rule. In this case, the vibrational energy of $27^{11}(113$ $\mathrm{cm}^{-1}$ ) is not enough to cause autodetachment, due to the relatively large binding energy of the DBS (146 \pm $\left.5 \mathrm{~cm}^{-1}\right)$ [1], as can be seen in Fig. 8. The vibrational energy of $26^{1}\left(150 \mathrm{~cm}^{-1}\right)$ may also be below the DBS binding energy, if all the error bars are considered. Nevertheless, the significantly enhanced $0_{0}^{0}$ peak is still surprising and should be caused by anharmonic effects, as will be shown more prominently in all the spectra shown in Fig. 6. 
In Figs. $4 \mathrm{~b}$ and 4c, which involve autodetachment from combinational levels of two modes, there are two significantly enhanced peaks, suggesting that the autodetachment rates of the two channels are comparable. Fig. 4b shows both peak $\mathbf{A}\left(19^{1}\right)$ and $\mathbf{B}\left(17^{1}\right)$ are enhanced, compared to peaks $0_{0}^{0}$ and C, indicating that both the $v_{17}$ and $v_{19}$ modes couple strongly with the DBS electron. Moreover, peak B is significantly stronger than peak $\mathbf{A}$, when comparing their intensity ratios in Fig. $4 \mathrm{~b}$ and Fig. 2b, suggesting that the coupling between the $v_{19}$ mode and the DBS electron is much stronger than that between the $v_{17}$ mode and the DBS electron.

Figs. $4 \mathrm{~d}-41$ all show that only one dominant neutral vibrational level is enhanced, that is, peak $\mathbf{g}\left(15^{1}\right)$ from $19^{11} 15^{\prime 1}$, peak $\mathbf{o}\left(17^{1} 16^{1}\right)$ from $19^{11} 17^{11} 16^{\prime 1}$, peak $\mathbf{l}\left(16^{2}\right)$ from $19^{\prime 1} 16^{\prime 2}$, peak $\mathbf{n}\left(24^{1} 16^{1}\right)$ from $19^{11} 16^{1} 24^{11}$, peak $\mathbf{C}\left(16^{1}\right)$ from $12^{11} 16^{11}$, peak o $\left(17^{1} 16^{1}\right)$ from $17^{\prime 2} 16^{1}$, peak $\mathbf{q}\left(19^{1} 26^{2} 23^{1}\right)$ from $19^{\prime 2} 26^{\prime 2} 23^{11}$, peak $\mathbf{C}\left(16^{1}\right)$ from $16^{11} 11^{1}$, and peak $\mathbf{j}\left(10^{1}\right)$ from $17^{11} 10^{1}$. This indicates that the coupling between the DBS electron and the other component vibrational mode is much more favored than that between the DBS electron and the mode(s) the final state represents. For example, in Fig. 4 d, peak $\mathbf{g}\left(15^{1}\right)$ is dominant due to autodetachment from the $19^{1} 15^{11}$ vibrational level of the DBS, while the $0_{0}^{0}$ and A peaks are from non-resonant electron detachment, as are also reflected in the PADs of the different peaks. Hence, the electron coupling between mode $v_{19}$ and the DBS electron is much stronger than that between mode $v_{15}$ and the DBS electron.

\subsection{Autodetachment from overlapping vibrational levels of the $D B S$}

In cases of autodetachment from overlapping vibrational levels of the DBS, multiple peaks can be enhanced from different autodetachment channels. If one of the overlapping level is an overtone of a single high-frequency mode, the $0_{0}^{0}$ peak should be significantly enhanced, as are the cases in Figs. 5a-5g. For example, the direct detachment peak B in Fig. 5a and peak A in Figs. 5b-5g serve as references to identify the enhancement of the $0_{0}^{0}$ peak in each case, in addition to the PAD. Furthermore, Figs. 5a-5c, $5 \mathrm{f}$ and $5 \mathrm{~g}$ all show one more enhanced peak, suggesting there is an overlapping combinational level preferentially autodetaching to one channel, similar to those observed in Figs. 4d-4l. Figs. 5d and 5e involve multiple overlapping levels with combinational modes, in addition to the fundamental level, 
yielding rather complicated resonant PE spectra. The assignments of all the observed peaks are given in Table 1. Figs. 5h-5k all correspond to the overlapping levels containing pure combinational modes, all giving more complicated resonant PE spectra. In each case, there are two or more enhanced vibrational peaks. Using the $\Delta \mathrm{v}=-1$ vibrational propensity rule and guided by the computed vibrational frequencies (Table 3), all the observed neutral vibrational levels and the vibrational level of the DBS, from which the autodetachment occurs, can be readily assigned, as given in Table 1.

It should be pointed out that the assignment of peak 27 in the photodetachment spectrum in Fig. 1 to the fundamental excitation of $11^{\prime 1}$ overlapping with the $24^{\prime 1} 16^{\prime 1}$ combinational level (Fig. 5c and Table 2) is different from that reported previously [1]. In ref. 1, peak 26 in the photodetachment spectrum was assigned to $11^{\prime 1}$. This prior assignment is clearly unreasonable, as can be seen from the resonant PE spectrum in Fig. 6f, where the $0_{0}^{0}$ peak is not really enhanced. The new assignment results in an improved vibrational frequency for $v_{11}$ (Table 3), which is consistent with the assignment of the resonant PE spectrum in Fig. $4 \mathrm{k}$, which also involves the $v_{11^{\prime}}$ mode $\left(16^{11} 11^{11}\right)$.

\subsection{The observation of the breakdown of the $\Delta v=-1$ propensity rule}

Since the $\Delta \mathrm{v}=-1$ propensity rule was derived under the harmonic approximation, it can be violated if there are significant anharmonic effects, allowing $\Delta \mathrm{v}=-2,-3, \ldots$ autodetachment to be observed [17]. The N1[U-H $]^{-}$anion is a large molecular species and anharmonic effects are expected, as already seen in Figs. 3a, 3b, and 4a. We have observed anharmonic effects in nine additional resonant PE spectra, as shown in Fig. 6, which all exhibit breakdown of the $\Delta v=-1$ propensity rule with autodetachment involving two or even three vibrational quanta. For example, in Fig. 6a, while the enhancement of peaks $0_{0}^{0}$ and $\mathbf{e}\left(25^{1}\right)$, corresponding to autodetachment from the $15^{\prime 1}$ and $25^{\prime 1} 19^{\prime 1}$ vibrational levels of the DBS, respectively, obey the $\Delta \mathrm{v}=-1$ propensity rule, the enhanced peak $\mathbf{b}\left(26^{1}\right)$ must come from autodetachment from the $26^{\prime 1} 27^{\prime 1} 18^{\prime 1}$ combinational level with two-quanta, $27^{\prime 1} 18^{\prime 1}$, coupled with the DBS electron. Similarly, the two enhanced peaks in Fig. $6 \mathrm{~b}$, peaks $0_{0}^{0}$ and $\mathbf{c}\left(27^{2}\right)$, corresponding to autodetachment from $21^{\prime 1}$ and $27^{\prime 2} 16^{\prime 1}$, respectively, obey the $\Delta \mathrm{v}=-1$ propensity rule, the other three enhanced peaks, peaks a $\left(27^{1}\right), \mathbf{C}\left(16^{1}\right)$ and $\mathbf{B}\left(17^{1}\right)$ are due to the autodetachment from $27^{\prime 2} 16^{\prime 1}$ and 
$27^{\prime 1} 26^{\prime 1} 17^{\prime 1}$ with two quanta $27^{\prime 2}, 27^{\prime 1} 16^{\prime 1}$ and $27^{\prime 1} 26^{\prime 1}$ coupled with the DBS electron, respectively. Moreover, the enhanced peak $\mathbf{C}\left(16^{1}\right)$ in Fig. 6c, peak $\mathbf{b}\left(26^{1}\right)$ and peak $\mathbf{C}\left(16^{1}\right)$ in Fig. 6 d, peak $\boldsymbol{\varepsilon}\left(27^{2} 26^{1}\right)$ in Fig. 6f and peak $\mathbf{k}\left(19^{2}\right)$ in Fig. 6i are all due to 2-quanta autodetachment from $27^{\prime 1} 26^{\prime 1} 16^{\prime 1}$, $27^{\prime} 26^{\prime 1} 24^{\prime 1} / 26^{\prime 2} 16^{\prime 1}, 27^{\prime 2} 26^{\prime 1} 19^{\prime 2}$ and $19^{\prime 2} 18^{\prime 1} 17^{\prime 1}$, respectively. In some cases, 3-quanta autodetachment is observed. In Fig. 6e, the enhanced peaks, such as peaks $\mathbf{a}\left(27^{1}\right)$ and $\mathbf{B}\left(17^{1}\right)$ are due to autodetachment from $27^{\prime 2} 26^{\prime 1} 17^{\prime 1}$ with three-quanta coupled with the DBS electron. Similarly, the enhanced peak $\mathbf{B}\left(17^{1}\right)$ in Fig. 6d, peak $\mathbf{t}\left(14^{1}\right)$ in Fig. $6 \mathrm{~g}$, and peak $\mathbf{i}\left(13^{1}\right)$ in Fig. 6h all correspond to 3-quanta autodetachment from $27^{\prime 3} 17^{\prime 1}, 27^{\prime 2} 26^{\prime 1} 14^{\prime \prime}$, and $27^{\prime 2} 26^{\prime 1} 13^{\prime 1}$, respectively.

\section{Conclusions}

In conclusion, we report both non-resonant and high-resolution resonant photoelectron imaging of cryogenically-cooled deprotonated uracil anion, N1[U-H] $]^{-}$. An excited dipole-bound state was observed previously near the detachment threshold. Resonant photoelectron spectra are obtained by tuning the detachment laser to the above-threshold vibrational levels of the dipole-bound state, resulting in highly non-Franck-Condon photoelectron spectra due to vibrational autodetachment. Many Franck-Condon inactive vibrational modes are observed, significantly expanding the capability of photoelectron spectroscopy. From both the resonant photoelectron spectra and the detachment spectrum, a total of 21 fundamental vibrational frequencies are obtained for the $\mathrm{N} 1[\mathrm{U}-\mathrm{H}] \bullet$ radical, including all eight lowfrequency out-of-plane modes, which are symmetry-forbidden in non-resonant photoelectron spectroscopy. The breakdown of the $\Delta \mathrm{v}=-1$ vibrational autodetachment propensity rule has been observed in many resonant photoelectron spectra, due to anharmonic effects. In particular, we have observed, in a number of resonant photoelectron spectra, intramolecular rescattering of the out-going electron, autodetached from specific vibrational levels of the dipole-bound state. The intramolecular rescattering effect can be considered as a half-collision between the outgoing electron and the neutral core, inducing excitation of low-frequency modes analogous to low-energy electron loss spectroscopy. Further theoretical study may be warranted to further understand vibronic coupling involving autodetachment dynamics from dipole-bound states and electron-molecule interactions. 
ACKNOWLEDGMENT. This work was supported by the National Science Foundation (CHE-1263745).

\section{REFERENCES}

[1] H.T. Liu, C.G. Ning, D.L. Huang, L.S. Wang, Angew. Chem. Int. Ed. 53 (2014) 2464-2468.

[2] T.A. Patterson, H. Hotop, A. Kasdan, D.W. Norcross, W.C. Lineberger, Phys. Rev. Lett. 32 (1974) 189-192.

[3] S.E. Novick, P.L. Jones, T.J. Mulloney, W.C. Lineberger, J. Chem. Phys. 70 (1979) 2210-2214.

[4] P.L. Jones, R.D. Mead, B.E. Kohler, S.D. Rosner, W.C. Lineberger, J. Chem. Phys. 73 (1980) 4419-4432.

[5] A.H. Zimmerman, J.I. Brauman, J. Chem. Phys. 66 (1977) 5823-5825.

[6] R.L. Jackson, A.H. Zimmerman, J.I. Brauman, J. Chem. Phys. 71 (1979) 2088-2094.

[7] R.L. Jackson, P.C. Hiberty, J.I. Brauman, J. Chem. Phys. 74 (1981) 3705-3712.

[8] K.R. Lykke, R.D. Mead, W.C. Lineberger, Phys. Rev. Lett. 52 (1984) 2221-2224.

[9] K.R. Lykke, K.K. Murray, D.M. Neumark, W.C. Lineberger, Phil. Trans. Math. Phys. Eng. Sci. 324 (1988) 179-196.

[10] C.G. Bailey, D.J. Lavrich, D. Serxner, M.A. Johnson, J. Chem. Phys. 105 (1996) 1807-1814.

[11] J. Schiedt, R. Weinkauf, J. Chem. Phys. 110 (1999) 304-314.

[12] I. Leon, Z. Yang, L.S. Wang, J. Chem. Phys. 139 (2013) 194306.

[13] H.T. Liu, C.G. Ning, D.L. Huang, P.D. Dau, L.S. Wang, Angew. Chem. Int. Ed. 52 (2013) 8976-8979.

[14] D.L. Huang, G.Z. Zhu, L.S. Wang, J. Chem. Phys. 142 (2015) 091103.

[15] D.L. Huang, H.T. Liu, C.G. Ning, L.S. Wang, J. Chem. Phys. 142 (2015) 124309.

[16] D.L. Huang, H.T. Liu, C.G. Ning, G.Z. Zhu, L.S. Wang, Chem Sci 6 (2015) 3129-3138.

[17] R.S. Berry, J. Chem. Phys. 45 (1966) 1228-1245.

[18] J. Simons, J. Am. Chem. Soc. 103 (1981) 3971-3976.

[19] D.L. Huang, H.T. Liu, C.G. Ning, L.S. Wang, J. Phys. Chem. Lett. 6 (2015) 2153-2157.

[20] L.S. Wang, J. Chem. Phys. 143 (2015) 040901.

[21] X.B. Wang, L.S. Wang, Rev. Sci. Instrum. 79 (2008) 073108.

[22] I. Leon, Z. Yang, H.T. Liu, L.S. Wang, Rev. Sci. Instrum. 85 (2014) 083106.

[23] G.A. Garcia, L. Nahon, I. Powis, Rev. Sci. Instrum. 75 (2004) 4989-4996.

[24] H.T. Liu, Y.L. Wang, X.G. Xiong, P.D. Dau, Z.A. Piazza, D.L. Huang, C.Q. Xu, J. Li, L.S. Wang, Chem. Sci. 3 (2012) 3286-3295.

[25] D.L. Huang, P.D. Dau, H.T. Liu, L.S. Wang, J. Chem. Phys. 140 (2014) 224315.

[26] J. Cooper, R.N. Zare, J. Chem. Phys. 48 (1968) 942; 49 (1968) 4252 (Erratum).

[27] A. Sanov, Ann. Rev. Phys. Chem. 65 (2014) 341-363.

[28] H. Ibach, Surf. Sci. 299 (1994) 116-128.

[29] A. Politano, G. Chiarello, G. Benedek, E.V. Chulkov, P.M. Echenique, Surf. Sci. Rep. 68 (2013) 305-389.

[30] P. Hockett, M. Staniforth, K.L. Reid, D. Townsend, Phys. Rev. Lett. 102 (2009) 253002.

[31] K. Regeta, M. Allan, Phys. Rev. Lett. 110 (2013) 203201. 